\title{
Aspergillus mulundensis sp. nov., a new species for the fungus producing the antifungal echinocandin lipopeptides, mulundocandins
}

\author{
Gerald F Bills ${ }^{1}$, Qun Yue ${ }^{1}$, Li Chen ${ }^{1}$, Yan $\mathrm{Li}^{1}$, Zhiqiang $\mathrm{An}^{1}$ and Jens C Frisvad ${ }^{2}$ \\ The invalidly published name Aspergillus sydowii var. mulundensis was proposed for a strain of Aspergillus that produced new \\ echinocandin metabolites designated as the mulundocadins. Reinvestigation of this strain (Y-30462 = DSMZ 5745) using \\ phylogenetic, morphological, and metabolic data indicated that it is a distinct and novel species of Aspergillus sect. Nidulantes. \\ The taxonomic novelty, Aspergillus mulundensis, is introduced for this historically important echinocandin-producing strain. The \\ closely related $\boldsymbol{A}$. nidulans FGSC A4 has one of the most extensively characterized secondary metabolomes of any filamentous \\ fungus. Comparison of the full-genome sequences of DSMZ 5745 and FGSC A4 indicated that the two strains share \\ 33 secondary metabolite biosynthetic gene clusters. These shared gene clusters represent $\sim 45 \%$ of the total secondary \\ metabolome of each strain, thus indicating a high level intraspecific divergence in terms of secondary metabolism. \\ The Journal of Antibiotics (2016) 69, 141-148; doi:10.1038/ja.2015.105; published online 14 October 2015
}

\section{INTRODUCTION}

Mulundocandin and deoxymulundocandin (Figure 1: 1, 2) are lipohexapeptides and potent antifungal antibiotics of the echinocandin class. ${ }^{1-3}$ Biosynthetically, they are closely related to echinocandin $\mathrm{B}$ and $\mathrm{C}$ (Figure 1: 3, 4) but differ by the inclusion of serine instead of threonine in the fifth position of the hexapeptide core and by a 12-methyl myristoyl side chain instead of a lineolyl side chain. Mulundocandin and its deshydroxy C4 homotyrosine form have been investigated extensively as potential lead structures for the development of echinocandin-type antifungal drugs. ${ }^{3-6}$ The structure elucidation of mulundocandin and its potent antifungal activity against Candida albicans, Cercospora beticola and Microsporum gypseum were described in 1987 from a strain of Aspergillus (Y-30462 = DSMZ 5745) isolated at Hoechst India Ltd located in Mulund district of Mumbai, India from a soil sample collected in Bangladesh. ${ }^{1,2}$ In the original publication, the fungus was considered to be an unusual variant of Aspergillus sydowii because of the presence of abundant Hülle cells and was designated as A. sydowii var. mulundensis. However, the name was published as a nomen nudum because no type specimen was designated and no Latin diagnosis was provided. During the course of development of a transformation method for the mulundocandinproducing strain by gene inactivation with the hygromycin resistance gene, the internal transcribed spacer ribosomal DNA of a subculture derived from DSMZ 5745 was sequenced (AJ312221), and the sequence data indicated the fungus was more closely related to A. nidulans than to A. sydowii. ${ }^{7}$
Recently, the full spectrum of echinocandin-type biosynthetic gene clusters, including the gene clusters encoding the starting molecules for the antifungal drugs, caspofungin, micafungin and anidulifungin, was investigated to determine the evolutionary origins and relationships of the echinocandins and to better understand the chemical logic underpinning the biosynthesis of this class of potent antifungal metabolites. $^{8,9}$ Strain Y-30462 was purchased from the DSMZ patent collection (DSMZ 5745), and its genomic DNA was extracted and used for genomic sequencing to elucidate the mulundocandin biosynthetic gene cluster and to search for additional biosynthetic genes that might be associated with mulundocandin biosynthesis and regulation. ${ }^{9}$ Sequencing the genome of DSMZ 5745 afforded us access to ribosomal and protein-encoding genes that could be used as phylogenetic markers. New sequence data and morphology confirmed that strain DSMZ 5745 was more closely allied to species in the A. nidulans group in Aspergillus sect. Nidulantes than to species in the sect. Versicolores (A. sydowii-A. versicolor group). Inclusion of strain DSMZ 5745 in sect. Nidulantes would be consistent with previous observations that multiple species and strains in sect. Nidulantes can produce echinocandins. ${ }^{10,11}$

The objective of this report was to clarify the name of the mulundocandin-producing strain and determine whether it was one of the known taxa in the species complex surrounding A. nidulans or whether it might represent a new species. The hypothesis was tested by probing current databases with ribosomal DNA sequences for this strain and by resampling available phylogenetic marker sequences to

\footnotetext{
${ }^{1}$ Texas Therapeutics Institute, The Brown Foundation Institute of Molecular Medicine, University of Texas Health Science Centre at Houston, Houston, TX, USA and ${ }^{2}$ Center for Microbial Biotechnology, Department of Systems Biology, Technical University of Denmark, Lyngby, Denmark

Correspondence: Dr GF Bills, Texas Therapeutics Institute, The Brown Foundation Institute of Molecular Medicine, University of Texas Health Science Center at Houston, 1881 East Road, 3SCR6.4676, Houston, TX 77054, USA.

E-mail: billsge@vt.edu or gerald.f.bills@uth.tmc.edu

Received 10 August 2015; revised 5 September 2015; accepted 11 September 2015; published online 14 October 2015
} 


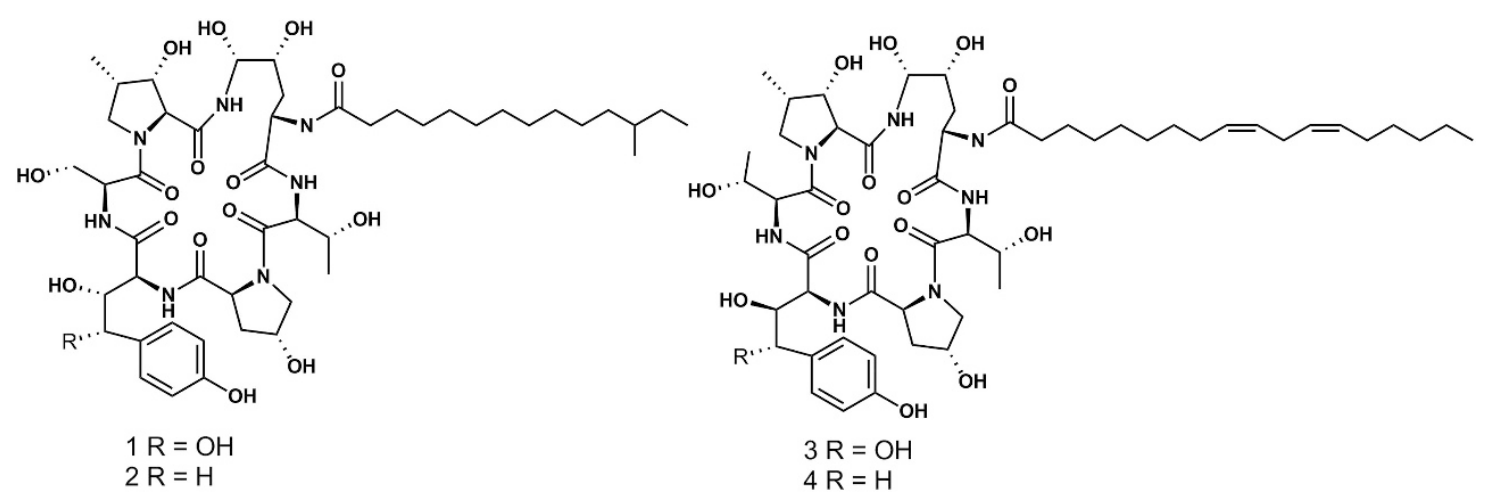

Figure 1 Chemical structures of Aspergillus echinocandins. Mundundocandin (1), Dexoxymulundocandin (2), Echinocandin B (3), Echinocandin C (4).

build multi-gene phylogenies. We also examined this question by evaluation of the strain's morphological characteristics and extrolite profiles. At last, the availability of a full-genome sequence for the mulundocandin-producing strain gave us an opportunity to supplement chemotaxonomic data with measurements of the potential divergence in secondary metabolism between two genome-sequenced species within Sect. Nidulantes. Therefore, we compared the genomic sequence of strain DSMZ 5745 with a strain of the central species of the A. nidulans complex, that of A. nidulans FGSC A4, probably the most studied secondary metabolome available from any fungus,. ${ }^{12-15}$ These data provide one of the first examples of using secondary metabolite gene clusters from sequenced genomes to aid in delimiting a new species and lead to the conclusion that the mulundocandinproducing strain represents a previously unrecognized species in the Aspergillus sect. Nidulantes.

\section{MATERIALS AND METHODS}

\section{Strain and morphological characterization}

The sole strain of A. sydowii var. mulundensis Y-30462 deposited as DSMZ $5745^{16}$ was purchased from the patent collection at the DSMZ (Deutsche Sammlung von Mikroorganismen und Zellkulturen). The strain was inoculated on Czapek yeast autolysate agar (CYA agar), malt extract agar (MEA) (Blakeslee formula, with Difco malt extract and peptone), MEA using the Oxoid formula (MEAOx), yeast extract sucrose agar, oat meal agar (from organic oat meal) and creatine sucrose agar and all were incubated at $25^{\circ} \mathrm{C} .{ }^{17}$ Furthermore, the strain was incubated at $37^{\circ} \mathrm{C}$ on CYA agar. Photomicrographs were taken from cultures grown on cornmeal agar at room temperature.

\section{Molecular phylogenetic analysis}

To clarify the phylogenetic status of A. sydowii var. mulundensis DSMZ 5745, we constructed a phylogenetic tree from a data set of the concatenated DNA fragments of the actin, calmodulin and $\beta$-tubulin genes that were resampled from a recent analysis of the A. nidulans species complex. ${ }^{18}$ The actin gene of DSMZ 5745 was amplified using primer pairs ACT-512F and ACT-783R, ${ }^{19}$ and other genes were extracted from the sequenced and annotated genome of DSMZ 5745. ${ }^{9}$ These sequences have been accessioned to Genbank (KP985733, KP985734, KP985735). The concatenated gene sequences for each species were aligned with ClustalW implemented in MEGA $6.0,{ }^{20}$ and analyzed by the maximum likelihood method based on a $\mathrm{K} 2+\mathrm{G}+\mathrm{I}$ model using MEGA 6.0. The data matrix and tree were accessioned to TreeBASE (www.treebase.org) as study 17905 .

In addition to the sequences mentioned above, the nuclear ribosomal internal transcribed spacer and large subunit were amplified and sequenced and accessioned to GenBank (KP985732).

\section{Extrolite analysis}

For extrolite analysis, three agar plugs were taken from CYA, MEA, yeast extract sucrose, OAT and MEAOx and extracted using established methods. ${ }^{21}$ The extracts were analyzed using ultra high pressure liquid chromatographydiode array detector (UHPLC-DAD) as described previously. ${ }^{22}$

Furthermore, we approximated the original fermentation conditions used for production of deoxymulundocandin to verify that DSMZ 5745 was still able to produce mulundocandins. The strain was cultured on malt-yeast extract agar at $25^{\circ} \mathrm{C}$ for 7 days to produce conidia and mycelia. Agar cultures were cut into small pieces $\left(0.5 \mathrm{~cm}^{3}\right)$, and 15 pieces were inoculated into $50 \mathrm{ml}$ of SMYA medium (Bacto neopeptone $10 \mathrm{~g}$, maltose $40 \mathrm{~g}$, yeast extract $10 \mathrm{~g}$, agar $4 \mathrm{~g}$, deionized $\mathrm{H}_{2} \mathrm{O} 1000 \mathrm{ml}$ ) in a $250-\mathrm{ml}$ Erlenmeyer flask. This seed culture was incubated at $25^{\circ} \mathrm{C}$ on a rotary shaker at $220 \mathrm{rpm}$ for 3 days. A $10-\mathrm{ml}$ aliquot of the seed medium was inoculated into $100 \mathrm{ml}$ of production medium (NZ amine EKC (N4767 Sigma-Aldrich, St Louis, MO, USA) $3 \mathrm{~g}$, Bacto peptone $3 \mathrm{~g}$, glucose $10 \mathrm{~g}$, soluble starch $24 \mathrm{~g}$, yeast extract $5 \mathrm{~g}, \mathrm{CaCO}_{3} 4 \mathrm{~g}$, $\mathrm{MnCl}_{2} .4 \mathrm{H}_{2} \mathrm{O} 0.5 \mathrm{mg}, \mathrm{ZnSO}_{4} .7 \mathrm{H}_{2} \mathrm{O} 0.22 \mathrm{mg}, \mathrm{CaCl}_{2} 0.55 \mathrm{mg}, \mathrm{FeSO}_{4} .7 \mathrm{H}_{2} \mathrm{O}$ $0.5 \mathrm{mg}, \mathrm{CuSO}_{4} \cdot 5 \mathrm{H}_{2} \mathrm{O} 0.16 \mathrm{mg}, \mathrm{CoCl}_{2} \cdot 6 \mathrm{H}_{2} \mathrm{O} 0.16 \mathrm{mg}$, deionized $\mathrm{H}_{2} \mathrm{O} 1000 \mathrm{ml}$ ) in each of two $500-\mathrm{ml}$ Erlenmeyer flasks. Flasks were incubated at $25^{\circ} \mathrm{C}$ on a rotary shaker at $220 \mathrm{rpm}$ for $76 \mathrm{~h}$.

A culture from one flask was vacuum-filtered to separate mycelium and culture broth. Each was extracted with $100 \mathrm{ml}$ of ethyl acetate, respectively. In the second culture, $100 \mathrm{ml}$ of ethyl acetate was added directly to extract the mycelium and broth simultaneously. The organic phase was evaporated to dryness and redissolved in methanol $(\mathrm{MeOH})$ at $30 \mathrm{mg} \mathrm{ml}^{-1}$. Then $10 \mu \mathrm{l}$ of each dissolved extracts were injected for HPLC-DAD-mass spectrometry (HPLC-DAD-MS) analysis on a linear gradient of $10-90 \%$ acetonitrile in water (with $0.1 \%$ formic acid) for $28 \mathrm{~min}$ at a flow rate of $1 \mathrm{ml} \mathrm{min}^{-1}$ through an Agilent Zorbax Eclipse Plus $\mathrm{C}_{18}$ reverse phase column $(4.6 \times 150 \mathrm{~mm}, 5 \mu \mathrm{m})$.

\section{Verification and structural elucidation of mulundocandins}

After the LC-MS analysis, the crude extract $(0.4 \mathrm{~g})$ was fractionated with a reversed-phase $\mathrm{C}_{18}$ column $\left(10-100 \% \mathrm{MeOH}\right.$ in $\mathrm{H}_{2} \mathrm{O}$ over $25 \mathrm{~min}$; $40 \mathrm{ml} \mathrm{min}^{-1}$ ) coupled to a Grace Reveleris X2 flash chromatography system. Fractions ( $25 \mathrm{mg}$ ) eluted with 60 and 70\% $\mathrm{MeOH}$ were combined and further purified by semi-preparative reverse phase HPLC (Agilent Zorbax SB-C 18 column; $5 \mu \mathrm{m} ; 9.4 \times 250 \mathrm{~mm} ; 60 \%$ acetonitrile in $\mathrm{H}_{2} \mathrm{O}$ over $15 \mathrm{~min}$; $\left.2 \mathrm{ml} \mathrm{min}^{-1}\right)$ to afford $1\left(1.2 \mathrm{mg}, \mathrm{t}_{\mathrm{R}} 8.37 \mathrm{~min}\right)$ and $2\left(2.1 \mathrm{mg}, t_{\mathrm{R}} 10.23 \mathrm{~min}\right)$.

NMR data were collected on a Bruker $500 \mathrm{MHz}$ NMR equipped with a 5-mm triple resonance cryoprobe at $298 \mathrm{~K}$. Residual solvent signals were used as reference $\left(\mathrm{CD}_{3} \mathrm{OD}: \delta_{\mathrm{H}} 3.31 / \delta_{\mathrm{C}} 49.2\right)$. The high-resolution mass spectra for each compound were acquired with an Agilent 6520 Q-TOF system in the positive ionization mode. For Q-TOF/MS conditions, voltages of fragmentor and capillary were kept at 130 and $3500 \mathrm{~V}$, respectively. Nitrogen was provided as the nebulizing and drying gas. Temperature of the drying gas was kept at $300{ }^{\circ} \mathrm{C}$. The flow rate of the drying gas and the pressure of the nebulizer were $101 \mathrm{~min}^{-1}$ and $25 \mathrm{psi}$, respectively. Full-scan spectra were acquired over a scan range of $\mathrm{m} / \mathrm{z} \quad 80-1500$ at 1.03 spectra $\mathrm{s}^{-1}$. The ${ }^{1} \mathrm{H}$ NMR and the 
high-resolution mass data for mulundocandin (1) and deoxymulundocandin (2) were consistent with previous literature values. ${ }^{1,3}$

\section{Comparison of secondary metabolomes of DSMZ 5745 and $A$. nidulans FGSC A4}

The repeat-masked nucleotide scaffolds and predicted proteins of Aspergillus mulundensis DSMZ 5745 were analyzed with anti-SMASH, ${ }^{23}$ employing the default parameters to identify the core secondary metabolite genes and to approximately delimit biosynthetic gene clusters. Previously determined core genes and gene clusters from $A$. nidulans were used as references sequences to basic local alignment search tool (BLAST) against the A. mulundensis genome to manually locate orthologous gene clusters. ${ }^{12,24}$ Orthologous gene clusters between $A$. mulundensis and $A$. nidulans were aligned and illustrated using Easyfig (Supplementary Figure S1) to determine gene identity and microsynteny. 25

\section{RESULTS}

\section{Phylogenetic analysis}

Basic local alignment search tool searches with internal transcribed spacer and large subunit sequences of DSMZ 5745 consistently retrieved high scoring similarities for various species of Aspergillus sect. Nidulantes, including A. falconensis, A. similis, A. multicolor, A. omanensis, A. navahoensis and A. fruticans; however, no particular species provided an unequivocal similarity match. Curiously, the closest match for an environmental DNA sequence using the internal transcribed spacer region was uncultured fungus clone 06CI5ID11 (KP243146, 96\% identity), thus indicating the species is not commonly encountered during metagenomic pyrosequencing or cloning experiments. We attempted to identify strain DSMZ 5745 and associate it with a previously described species of Aspergillus sect. Nidulantes. We reconstructed the data matrix from a recent phylogenetic investigation of Aspergillus sect Nidulantes ${ }^{18}$ using the concatenated actin, calmodulin and $\beta$-tubulin gene sequences and tested whether the corresponding sequences from DSMZ 5745 would cluster unambiguously with one of the known species. Tree topology resulting from maximum likelihood analysis closely tracked the previous analysis (Figure 2). Sequences for DSMZ 5745 were intercalated deeply in a basal branch of the tree, in a strongly supported, but isolated branch with A. pluriseminatus (Figure 2). Its location on this isolated node was consistent with the hypothesis that DSMZ 5745 was a previously unrecognized species. The relatively isolated position among the other species and strains in the tree was consistent with the chemical uniqueness of the strain based on extrolite and genomic analysis (Supplementary Table S1), and led to the conclusion that the strain represented a previously unrecognized species.

\section{Taxonomy}

Aspergillus mulundensis Bills and Frisvad sp. nov. MycoBank MB813062

三 Aspergillus sydowii var. mulundensis, Journal of Antibiotics 40:275, 1987, nomen nudum

Typus: DSMZ $5745^{\mathrm{T}}$ patent collection; IBT $33104^{\mathrm{T}}$ (Figure $3 \mathrm{a}-\mathrm{i}$ ), ex soil, Bangladesh

Etymology: from Mulund, Mumbai, India, site of the former headquarters of Hoechst India Ltd, where the fungus was isolated and first recognized to produce a new echinocandin-type antibiotic, mulundocandin.

Diagnosis: A. mulundensis differs from A. nidulans by producing smaller penicillate heads, shorter conidiophore stipes, by growing more slowly on all media, especially at $37^{\circ} \mathrm{C}$, and by not producing ascomata. It differs from $A$. sydowii by having metulae only covering the upper $50 \%$ of the conidial heads, by having dark dull green conidia, and by not growing well on creatine sucrose agar. Hülle cells were not observed. The A. mulundensis genome harbors the mulundocandin gene cluster that is absent in A. nidulans, but it lacks genes for biosynthesis of sterigmatocystin and penicillin that are characteristic of $A$. nidulans.

Description: colonies on CYA. 1 week, $25^{\circ} \mathrm{C}, 20-22 \mathrm{~mm}$, poor sporulation, white mycelium and a yellow-brown reverse. Colonies on MEA, 1 week, $25^{\circ} \mathrm{C}, 7-15 \mathrm{~mm}$, no sporulation, white mycelium, and an orange-brown reverse, colonies on yeast extract sucrose agar 1 week, $25^{\circ} \mathrm{C}, 27-35 \mathrm{~mm}$, no sporulation, white mycelium, warm orange-brown reverse, colonies on oat meal weakly sporulating, whereas mycelium, cream-colored reverse. On CYA at $37^{\circ} \mathrm{C}$, colonies 3-9 $\mathrm{mm}$, no sporulation and white mycelium and cream-colored reverse. Conidia en masse colored dark dull green to dull blue-green, and produced best on MEA and oat meal agars after 2 weeks of incubation. Conidial heads radiate and globose. On creatine sucrose very poor growth, 5-16 mm diameter, and no acid production. Fully developed Aspergilli biseriate with metulae covering the upper $50-60 \%$ of the head, vesicle small, globose to pear shaped, $8-20 \mu \mathrm{m}$, metulae 5-7 $\mu \mathrm{m} \times 2-3 \mu \mathrm{m}$, cylindrical, each having $1-3$ phialides that are short, 5-8 $\mu \mathrm{m} \times 2.5-4 \mu \mathrm{m}$, with broad collula, stipes smooth- and thick-walled, uncolored to light brown, short, in some aspergilla $7-12 \mu \mathrm{m} \times 5-7 \mu \mathrm{m}$, others are longer $25-120 \mu \mathrm{m} \times 5-7.5 \mu \mathrm{m}$. Several single phialides and small penicillate heads are also produced, similar to those seen in $A$. sydowii and $A$. asperescens, ${ }^{26,27}$ especially on aerial hyphae. In some conidial heads, phialides are intermixed with metulae and phialides. Conidia globose, delicately spinulose to verrucose, 2.5-4.5 $\mu \mathrm{m}$ diameter. Ascomata and sclerotia not observed, not even in cultures grown on autoclaved rehydrated wheat seeds in ambient light for 1 month. Hülle cells were not observed, but fide Roy et al., ${ }^{2}$ 'Hülle cells are abundant, thick-walled, colorless at first becoming purple with age, scattered unevenly, 18.7-31.2 ×25-37.5 $\mu \mathrm{m}$ '.

The type strain (Figure 3a-i) can produce azaphilone extrolites, but they are not falconensins produced by the related Aspergillus falconensis, ${ }^{28-31}$ but rather are related to sclerotiorins with UV spectra identical to those in the extracts of Penicillium sclerotiorum. In addition, A. mulundensis produces extrolites with UV spectra suggesting karnatakafurans or similar dibenzofurans. ${ }^{32,33}$ The putative dibenzofuran had a UV spectrum with absorptions at $230 \mathrm{~nm}(100 \%)$, 265 (45\%), 287 (28\%), 303 (17\%), 314 (22\%). Finally, this strain continued to produce mulundocandin and deoxymulundocandin when grown on a medium similar to the Hoechst production medium; $;^{2,3,16}$ however, these extrolites were undetectable on the standardized agar media and extraction method used for extrolite characterization (Figure 4). As expected, the liquid fermentation extracts were strongly inhibitory to growth of C. albicans ATCC 10231 in an agar zone of inhibition assay (data not shown). Like the pneumocandins, mulundocandins were produced during exponential growth and were strongly associated with the mycelium and were not secreted in the culture broth (Figure 4).

\section{The predicted secondary metabolome of the A. mulundensis}

The mulundocandin gene cluster was recently delineated in A. mulundensis, ${ }^{9}$ and it was pointed out this strain shared in common with $A$. nidulans, the gene clusters for the lipopentapeptide, emericellamide and a two-module non-ribosomal peptide synthetases (NRPS) inp gene cluster that is not yet linked to a known metabolite. Analysis with Anti-SMASH and BLAST searches with key polyketide synthase and NRPS domains revealed a rich repertoire of secondary metabolite biosynthetic genes including 33 polyketide synthases (PKS), 


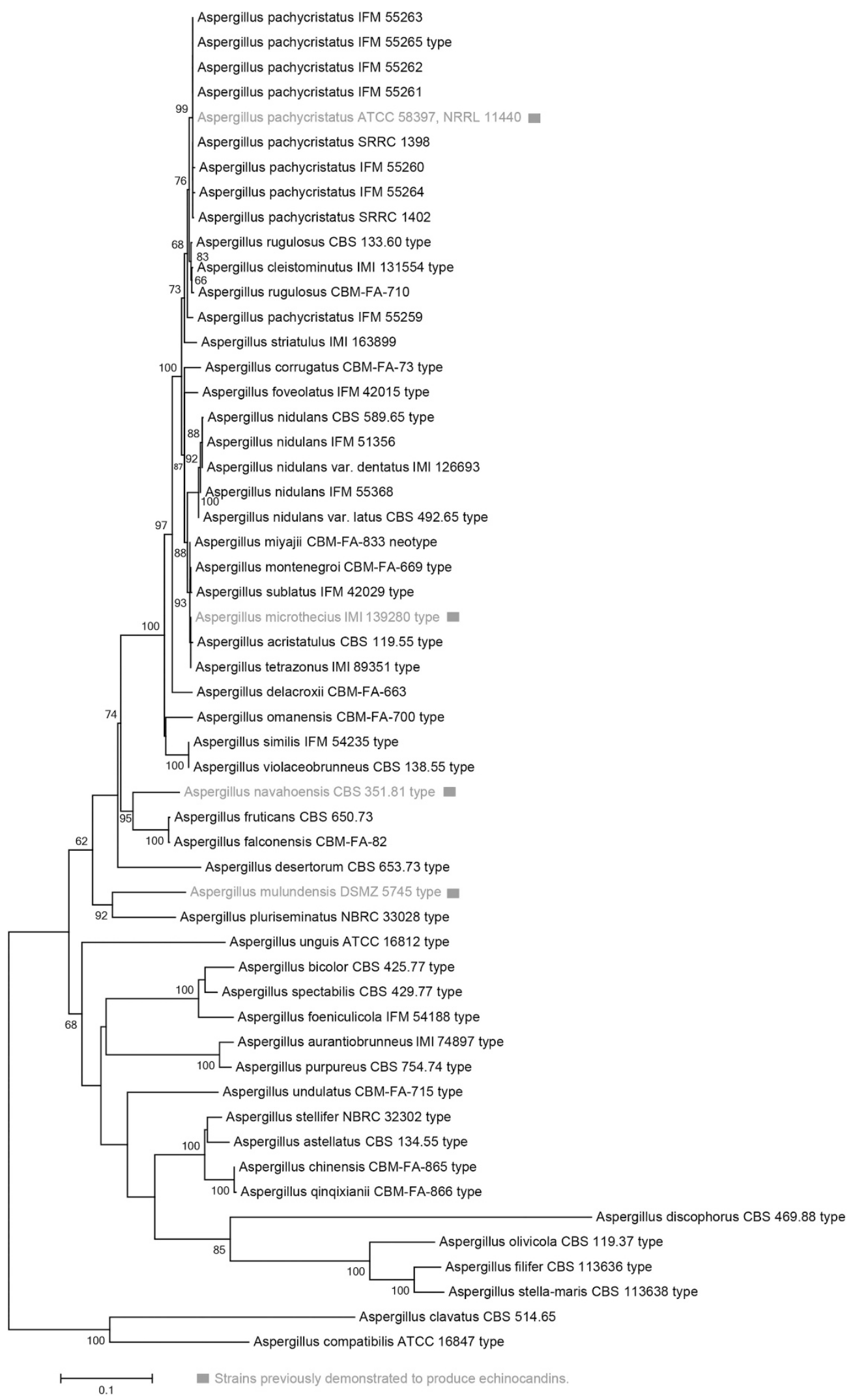

Figure 2 Maximum likelihood analysis of Aspergillus mulundensis DSMZ 5745 and selected fungi of Aspergillus sect. Nidulantes based on a combined three-gene data set including the DNA fragments of actin, calmodulin and $\beta$-tubulin genes. The evolutionary history was inferred by using the maximum likelihood method based on a $\mathrm{K} 2+\mathrm{G}+\mathrm{l}$ model. Branch lengths are proportional to the number of genetic changes. Numbers at branch points represent bootstrap values $>60 \%$ of 1000 bootstrap replications. The alignment data matrix consisted of 1795 characters and 54 strains. Strains in red with a red box have been verified to produce echinocandins. A full color version of this figure is available at The Journal of Antibiotics journal online. 

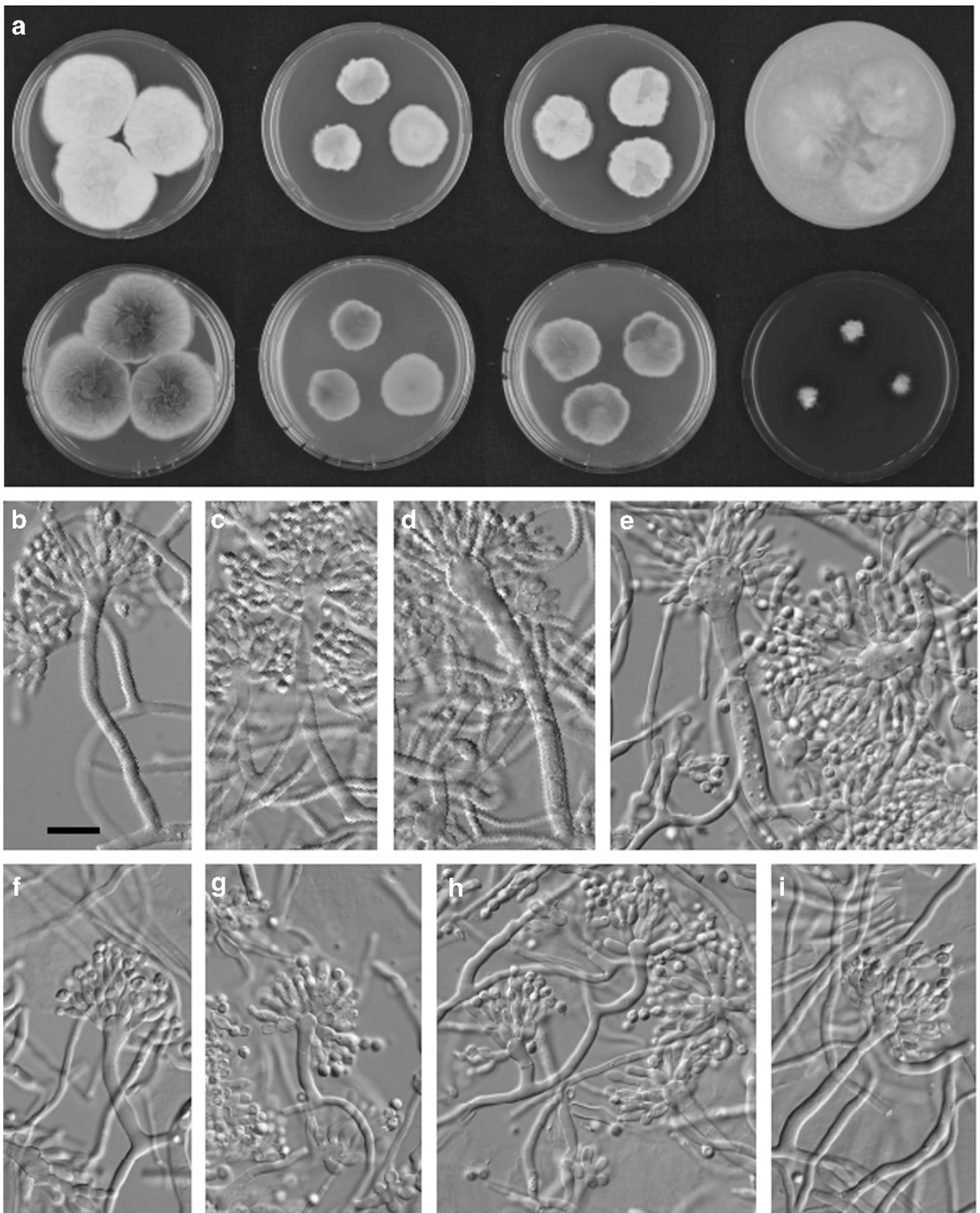

Figure 3 Aspergillus mulundensis, type strain DSMZ 5745=IBT 33104. (a) Seven-day old colonies. Top row, left to right, YES, MEA, CYA, OA; Bottom row YES reverse, MEA reverse, CYA reverse, CREA. (b-i) Conidiophores and conidia. All photographed at the same scale, scale bar in $B=10 \mu \mathrm{m}$. A full color version of this figure is available at The Journal of Antibiotics journal online.

19 NRPS, 10 terpene synthases and 5 dimethylallyl tryptophan synthases dispersed among 73 gene clusters (Figure 5). This estimate is similar to the $\sim 71$ core genes and gene clusters identified in $A$. nidulans FGSC A4. ${ }^{12}$ Secondary metabolite gene clusters previously identified in FGSC A4 were aligned to annotated scaffolds from DSMZ 5745 to verify that other secondary metabolite gene clusters not only shared significant sequence homology, but also were similar in microsynteny (Supplementary Figure 1). In total, 33 gene clusters were found to be highly orthologous (Figure 5) and to share significantly similar microsynteny (Supplementary Figure S1). Besides the presence of the mulundocandin and emericellamide gene clusters, an additional 9 of these 33 other gene clusters were highly similar to those in $A$. niduluans that have been linked to characterized compounds or compound families (Supplementary Figure S1). The analysis predicted that DSMZ 5745 should produce asperfuranones, ${ }^{34}$ alternariol or related isocoumarins, the non-ribosomal peptideterpenes nidulanin and fungisporin, ${ }^{12,24}$ orsellinaldehyde, orsellinic acid and derivatives, asperthecin, ${ }^{35}$ the siderophore ferricrocin ${ }^{36}$ and a green conidial pigment encoded by $w A$. However, most of the shared gene clusters are not yet linked with a known metabolite.

\section{DISCUSSION}

Aspergillus mulundensis originated from a soil sample from an unspecified location in Bangladesh. Based on our investigation, A.sydowii var. mulundensis should be considered a homotypic synonym because DSMZ 5745 was derived from Hoechst's strain Y-30462, and the name mulundensis should be retained as the species epithet to maintain continuity with the previous body of work on the 

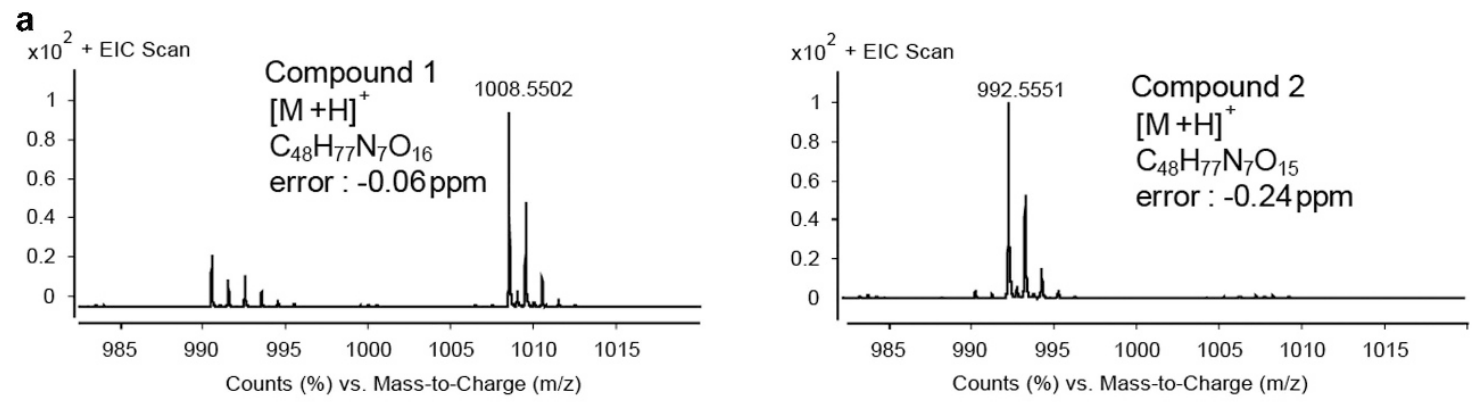

b x $10^{3}$ HPLC-UV (210 nm), Whole culture

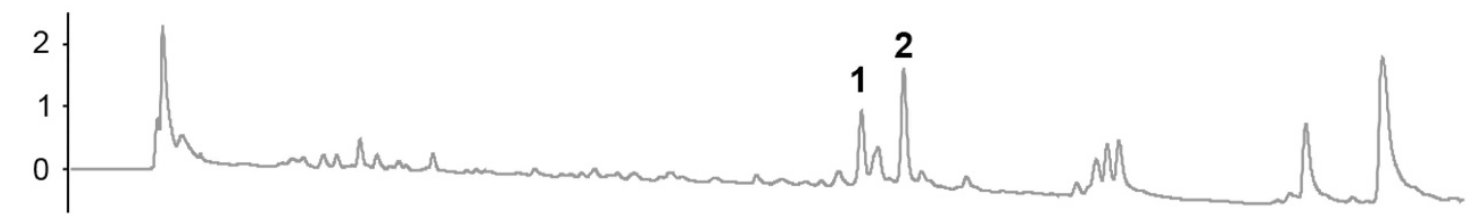

$\times 10^{3}$ HPLC-UV (210 nm), Mycelium only

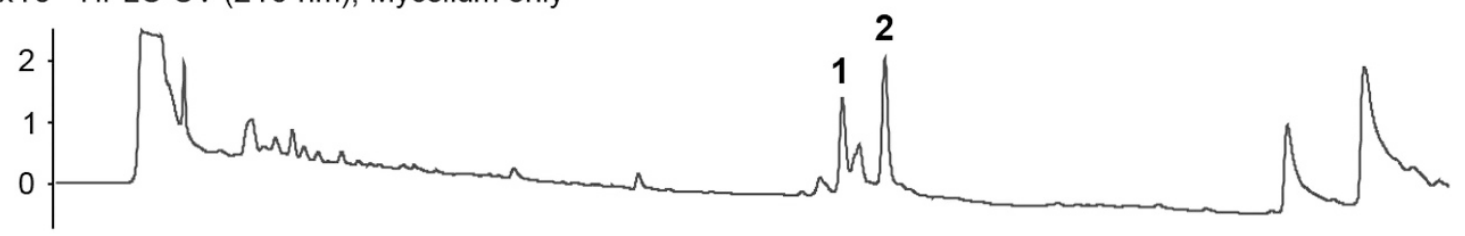

x10 3 HPLC-UV (210 nm), Broth only

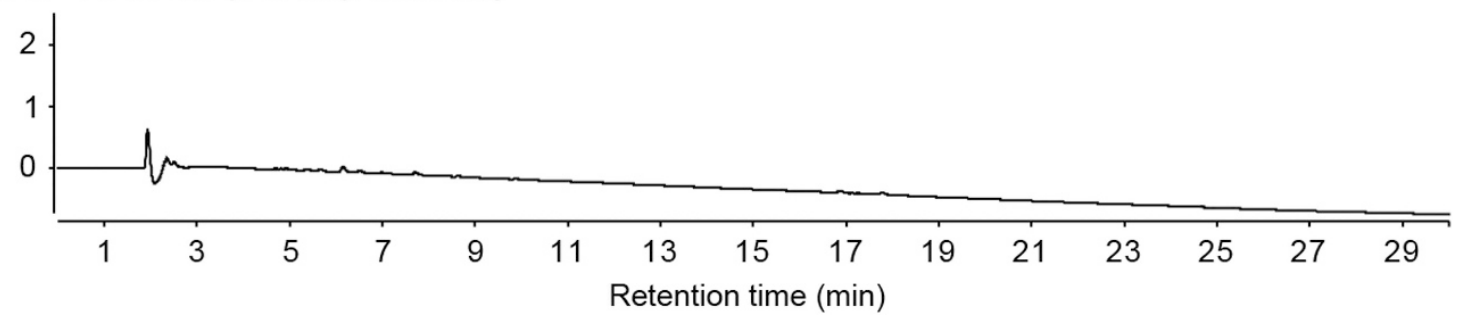

Figure 4 Identification of mulundocandins in fermentations of $A$. mulundensis DSMZ 5745. (a) Identification of mulundocandin (1) and deoxymulundocandin (2) by high-resolution MS. (b) HPLC UV chromatograms demonstrating that mulundocandins are localized in the mycelium and not excreted into the culture broth. See Material and methods for extraction and fermentation conditions. A full color version of this figure is available at The Journal of Antibiotics journal online.
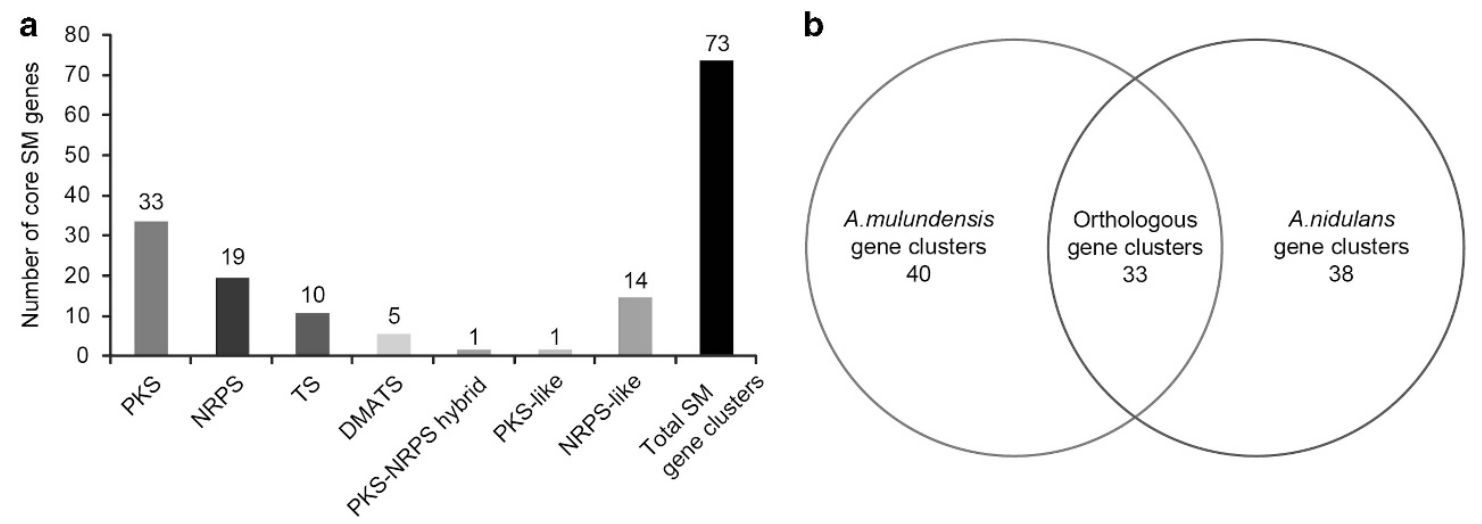

Figure 5 Analysis of secondary metabolome of $A$. mulundensis. (a) Numbers of core secondary metabolite gene clusters and core genes predicted by anti-SMASH. (b) Venn diagram comparison of secondary metabolomes of $A$. mulundensis and $A$. nidulans FGSC A4. PKS, polyketide synthase; NRPS, non-ribosomal peptide synthetase; TS, terpene synthase; DMATS, dimethylallyl tryptophan synthase. 
mulundocandin-producing fungus. The new species shares small penicillate heads with $A$. sydowii and $A$. asperescens, whereas it shares the general aspect of the aspergilla of A. nidulans. Aspergillus mulundensis shares Hülle cells with most other species in section Nidulantes. The greenish conidial pigments apparently have the same biogenic origin as those in A. nidulans (Supplementary Figure S1). We do not know the history of culture transfers and storage conditions of the type strain prior to its accession to the DSMZ. However, the apparent loss of the ability to form Hülle cells and the tendency of the strain to sector and grow as poorly sporulating and unpigmented mycelium suggest that the original wild-type isolate may have suffered excessive transfers or perhaps even may have been mutated to select for sub-strains with increased titers of mulundocandins. The DNA sequences, extrolites and gene clusters identified in this study should aid in the future recognition of new strains of $A$. mulundensis.

The type strain of $A$. mulundensis produces a unique combination of extrolites. It shares the dibenzofurans with $A$. multicolor, A. karnatakaensis, A. aeneus and echinocandin-like metabolites with A. pachycristatus, A. cleistominutus, A. microthecius, A. navahoensis, A. quadrilineatus and A. rugulosus. However, among the echinocandins described to date from Aspergilli, mulundocandin is unique because of its combination of a 12-methymyristoyl side and serine in the fifth position of the core peptide. Comparison of its genome with that of A. nidulans FGSC A4 also predicts the type strain can produce emericellamides, asperfuranones alternariol or related isocoumarins, nidulanins and fungisporins, orsellinaldehyde, orsellinic acid and derivatives, asperthecin and the siderophore ferricrocin. Aspergillus mulundensis also shares the emericellamide, asperthecin and ferricrocin gene clusters with A. ustus of subgenus Nidulantes, Sect. Usti. $^{37}$ Also significantly, A. mulundensis lacks the genetic machinery to produce sterigmatocystin, penicillins and several other prominent extrolites characteristic of $A$. nidulans.

It is worth pointing out that the standardized method for extrolite production and extraction from agar plugs $^{17}$ did not detect mulundocandins, yet they were readily produced using an approximation of fermentation conditions published in a previous patent. ${ }^{16}$ Our analysis of the separated culture broth and mycelium indicated that mulundocandins were only present in the mycelium and not in the liquid (Figure 4), a pattern also observed in the pneumocandins, thus, suggesting that echinocandin metabolites are stored intracellularly and not secreted. This pattern of mycelial associated metabolite sequestration is also characteristic many other fungal metabolites. ${ }^{38-40}$ Thus, considering the potential biological importance of the echinocandins to the fungi that produce them and that several species in sect. Nidulantes are known to readily produce echinocandins, future comprehensive analysis of sect. Nidulantes should also include methods specifically to detect whether strains produce echinocandins or not, either by using previously described chemotyping and bioassay methods, ${ }^{10,41}$ or by using a genetic method that would specifically amplify sequences unique to echinocandin NRPS genes.

\section{CONFLICT OF INTEREST}

The authors declare no conflict of interest.

\section{ACKNOWLEDGEMENTS}

We thank Vera Weihs (DSMZ) for assistance in obtaining strain DSMZ 5745. This work was supported by the University of Texas Health Science Center at Houston new faculty start-up funds and the Kay and Ben Fortson Endowment to GFB, and the National Natural Science Foundation of China (Grant 31328001), Texas Emerging Technology Fund, and the Welch Foundation (Grant No. AU00024) to ZA.
1 Mukhopadhyay, T, Ganguli, B. N, Fehlhaber, H. W, Kogler, H \& Vertesy, L. Mulundocandin, a new lipopeptide antibiotic. II. Structure elucidation. J. Antibiotics 40, 281-289 (1987).

2 Roy, K, Mukhopadhyay, T, Reddy, G. C. S, Desikan, K. R \& Ganguli, B. N. Mulundocandin, a new lipopeptide antibiotic. I. Taxonomy, fermentation, isolation and characterization. J. Antibiotics 40, 275-280 (1987).

3 Mukhopadhyay, $\mathrm{T}$ et al. Deoxymulundocandin - A new echinocandin type antifungal antibiotic. J. Antibiotics 45, 618-623 (1992).

4 Lal, B, Gund, V. G, Bhise, N. B \& Gangopadhyay, A. K. Mannich reaction: an approach for the synthesis of water soluble mulundocandin analogues. Biorg. Med. Chem. 12, 1751-1768 (2004)

5 Lal, B et al. Semisynthetic modifications of hemiaminal function at ornithine unit of mulundocandin, towards chemical stability and antifungal activity. Biorg. Med. Chem. 11, 5189-5198 (2003).

6 Hawser, S, Borgonovi, M, Markus, A \& Isert, D. Mulundocandin, an echinocandin-like lipopeptide antifungal agent: biological activities in vitro. J. Antibiotics 52, 305-310 (1999).

7 Schmitt, E. K, Eilinghoff, B, Olliger, R, Decker, H \& Kuck, U. Development of molecular tools for the mulundocandin producer Aspergillus sydowii: DNA-mediated transformation and reporter gene expression. Appl. Microbiol. Biotechnol. 58, 625-631 (2002).

8 Bills, G et al. New insights into the echinocandins and other fungal non-ribosomal peptides and peptaibiotics. Nat. Prod. Rep. 31, 1348-1375 (2014).

9 Yue, $Q$ et al. Evolution of chemical diversity in the echinocandin lipopeptide antifunga metabolites. Euk. Cell 14, 698-718 (2015).

10 de la Cruz, $M$ et al. Chemical and physical modulation of antibiotic activity in Emericella species. Chem. Biodivers. 9, 1095-1113 (2012)

11 Dreyfuss, M. M. Neue Erkenntnisse aus einem pharmakologischen Pilz-screening. Sydowia 39, 22-36 (1986).

12 Andersen, M. R et al. Accurate prediction of secondary metabolite gene clusters in filamentous fungi. Proc. Natl Acad. Sci. USA. 110, E99-E107 (2013).

13 Bergmann, S et al. Genomics-driven discovery of PKS-NRPS hybrid metabolites from Aspergillus nidulans. Nat. Chem. Biol. 3, 213-217 (2007).

14 Palmer, J. M et al. Secondary metabolism and development is mediated by LImF control of VeA subcellular localization in Aspergillus nidulans. PLoS Genet. 9, e1003193 (2013)

15 von Döhren, H. A survey of nonribosomal peptide synthetase (NRPS) genes in Aspergillus nidulans. Fungal Gen. Biol 46, S45-S52 (2009).

16 Roy, K, Mukhopadhyay, T, Fehlhaber, H. W, Kogler, H \& Ganguli, B. N. Antibiotic, deoxymulundocandin, a process for its production and its use as a medicament. US Patent 5,387,670 (1995).

17 Samson, R. A, Houbraken, J, Thrane, U, Frisvad, J. C \& Andersen, B. Food and Indoor Fungi. CBS laboratory manual Series 2, CBS KNAW Fungal Biodiversity Center, Utrecht, NL (2010).

18 Matsuzawa, $\mathrm{T}$ et al. The correlation among molecular phylogenetics, morphological data, and growth temperature of the genus Emericella, and a new species. Mycoscience 53, 433-445 (2012).

19 Carbone, I \& Kohn, L. M. A method for designing primer sets for speciation studies in filamentous ascomycetes. Mycologia 91, 553-556 (1999).

20 Tamura, K, Stecher, G, Peterson, D, Filipski, A \& Kumar, S. MEGA6: Molecular evolutionary genetics analysis version 6.0. Mol. Biol. Evol. 30, 2725-2729 (2013).

21 Smedsgaard, J. Micro-scale extraction procedure for standardized screening of fungal metabolite production in cultures. J. Chromatogr. A. 760, 264-270 (1997).

22 Klitgaard, A et al. Aggressive dereplication using UHPLC-DAD-QTOF: screening extracts for up to 3000 fungal secondary metabolites. Analyt. Bioanalyt. Chem. 406 1933-1943 (2014).

23 Weber, T et al. antiSMASH 3.0 - a comprehensive resource for the genome mining of biosynthetic gene clusters. Nucl. Acids Res. 43, W237-W243 (2015)

24 Klitgaard, A, Nielsen, J. B, Frandsen, R. J. N, Andersen, M. R \& Nielsen, K. F. Combining stable isotope labeling and molecular networking for biosynthetic pathway characterization. Anal. Chem. 87, 6520-6526 (2015).

25 Sullivan, M. J, Petty, N. K \& Beatson, S. A. Easyfig: a genome comparison visualizer. Bioinformatics 27, 1009-1010 (2011).

26 Thom, C \& Raper, K. B. A Manual of the Aspergilli, Bailliére Tindall \& Cox, London, UK (1945).

27 Stolk. A. C. Aspergillus asperescens n. sp. Antonie van Leeuwenhoek 20, 299-304 (1954).

28 Itabashi, T et al. and D, new compounds related to azaphilone, from Emericella falconensis. Chem. Pharm. Bull. 40, 3142-3144 (1992).

29 Itabashi, T, Ogasawara, N, Nozawa, K \& Kawai, K.-i. Isolation and atructures of new azaphilone derivatives, falconensins E-G, from Emericella falconensis and absolute configurations of falconensins A-G. Chem. Pharm. Bull. 44, 2213-2217 (1996).

30 Itabashi, T, Nozawa, K, Nakajima, S \& Kawai, K.-i. A new azaphilone, falconensin H, from Emericella falconensis. Chem. Pharm. Bull. 41, 2040-2041 (1993).

31 Ogasawara, N \& Kawai, K.-I. Hydrogenated azaphilones from Emericella falconensis and E. fruticulosa. Phytochemistry 47, 1131-1135 (1998).

32 Manniche, $\mathrm{S}$ et al. Karnatakafurans A and B: Two dibenzofurans isolated from the fungus Aspergillus karnatakaensis. J. Nat. Prod. 67, 2111-2112 (2004).

33 Rateb, M. E et al. Dibenzofurans from the marine sponge-derived ascomycete Super1F1-09. Botanica Marina 53, 499-506 (2010).

34 Chiang, Y.-M et al. A gene cluster containing two fungal polyketide synthases encodes the biosynthetic pathway for a polyketide, asperfuranone, in Aspergillus nidulans. J. Am. Chem. Soc. 131, 2965-2970 (2009). 
35 Szewczyk, E et al. Identification and characterization of the asperthecin gene cluster of Aspergillus nidulans. Appl. Environ. Microbiol. 74, 7607-7612 (2008).

36 Eisendle, $\mathrm{M}$ et al. The intracellular siderophore ferricrocin is involved in iron storage oxidative-stress resistance, germination, and sexual development in Aspergillus nidulans. Euk. Cell 5, 1596-1603 (2006).

$37 \mathrm{Pi}, \mathrm{B}$ et al. A genomics based discovery of secondary metabolite biosynthetic gene clusters in Aspergillus ustus. PLOS ONE 10, e0116089 (2015).

38 Hegge, A, Lønborg, R, Nielsen, D \& Sørensen, J. Factors influencing production of fusaristatin A in Fusarium graminearum. Metabolites 5, 184-191 (2015).
39 Droce, A, Sørensen, J. L, Giese, H \& Sondergaard, T. E. Glass bead cultivation of fungi: Combining the best of liquid and agar media. J. Microbiol. Meth. 94, 343-346 (2013).

40 Bills, G. F, Dombrowski, A. W \& Goetz, M. A. The 'FERMEX' method for metabolite-enriched fungal extracts. Methods Mol. Biol. 944, 79-96 (2012)

41 Bills, G et al. Enhancement of antibiotic and secondary metabolite detection from filamentous fungi by growth on nutritional arrays. J. Appl. Microbiol. 104, 1644-1658 (2008)

Supplementary Information accompanies the paper on The Journal of Antibiotics website (http://www.nature.com/ja) 